\title{
The Future of Epigenetics: Development of Technologies and Creation of Clinical Markers Predictive of Maternal Perinatal Risk
}

\section{El futuro de la Epigenética: Desarollo de Tec- nologías y Creación de Marcadores Clínicos Predictivos de Riesgo Materno Perinatal}

XVIII International Seminar on Health, Food and Human Nutrition

Corresponding Author:

Iván Enrique Naranjo Logroño naranjometropolitana@hotmail.com

Published: 9 September 2021

Production and Hosting by Knowledge E

(c) Iván Enrique Naranjo Logroño et al. This article is distributed under the terms of the Creative Commons Attribution License, which permits unrestricted use and redistribution provided that the original author and source are credited.
Iván Enrique Naranjo Logroño ${ }^{1}$, Anthony Alfonso Naranjo Coronel ${ }^{2}$, Cynthia Johana Navarro Rivadeneira ${ }^{1}$, and Carla Victoria Sandoval Flores ${ }^{1}$

${ }^{1}$ Carrera de Medicina, Facultad de Salud Pública, ESPOCH, Riobamba, Chimborazo, Ecuador

${ }^{2}$ Médico Cirujano, COLPOMED Centro, Hospital del Día, Riobamba, Chimborazo, Ecuador

\section{Abstract}

Introduction: Epigenetics is the study of the interaction between genotype and phenotype, first described by Waddingtonen in 1939. Objective: To identify new technologies that predict clinical markers of perinatal maternal risk with the help of epigenetics. Method: We searched all the databases that have had an impact worldwide, including Elsevier, WoS, Pubmed, Scielo, Redalyc, among others, generating a search with the terms epigenetic, clinical markers. Results: Of a total of 37 preselected articles, three were discarded, selecting 34 articles that met the inclusion/exclusion criteria in all that covers the topic and objective of this research work. Discussion: There are new lines of research that allow to know the epigenome of each individual and how the environment interact on it. Epigenetic biomarkers that exceed the usual diagnostic techniques on different pathologies are being developed. It is very important to continue in the search for information, for this reason several countries that have the appropriate technology are conducting epigenetic studies for later use in medicine. Conclusion: The woman during pregnancy experiences a series of changes that predispose her to the development of some pathology and the research in them is limited by her condition, but the diseases that stand out are diabetes, obesity and preeclampsia where epigenetics has shown that changes in specific genes occur.

\section{Resumen}

Introducción: La epigenética es el estudio de la interacción entre el genotipo y el fenotipo, descrito por primera vez por Waddingtonen en 1939. Objetivo: Identificar nuevas tecnologías que pronostiquen marcadores clínicos de riesgo materno perinatal con ayuda de la epigenética. Método: Se procedió a realizar búsquedas en todas las bases de datos que a nivel mundial han tomado impacto, entre ellas están Elsevier, WoS, Pubmed, Scielo, Redalyc, entre otros, generando búsqueda con los términos epigenética, marcadores clínicos. Resultados: De un total de 37 artículos preseleccionados, se descartó tres, seleccionando 34 artículos que cumplían con los criterios de inclusión/exclusión en todo lo que abarca el tema y el objetivo de este trabajo investigativo. Discusión: Existen nuevas líneas de investigación que permiten conocer el epigenoma de cada individuo y como el ambiente influye sobre este. Se están 
desarrollando biomarcadores epigenéticos que superen las técnicas habituales de diagnóstico para la detección de diversas patologías. Es muy importante seguir en la búsqueda de información, por esto varios países que cuentan con la tecnología adecuada se encuentran realizando estudios de epigenética para su uso posterior en la medicina. Conclusión: La mujer durante el embarazo experimenta una serie de cambios que le predisponen al desarrollo de alguna patología y las investigaciones en ellas se ven limitadas por su condición, pero las enfermedades que resaltan son la diabetes, obesidad y la preeclampsia donde la epigenética ha demostrado que suceden cambios en genes específicos.

Palabras Clave: epigenética, genoma humano, biomarcadores.

\section{Introducción}

La epigenética estudia complejos procesos que existen entre el genotipo y el fenotipo, explicando por qué algunos organismos vivos expresan unos genes y silencian otros; descrito por primera vez por Waddingtonen en 1939. Antes de que tome relevancia el término epigenética, se tomaba en cuenta que la relación entre los genes y el ambiente se producía por un determinismo genético que también plantea que nuestras características dependen completamente de nuestros genes. Waddingtonen trató de demostrar que ambas disciplinas están ligadas y son necesarias para explicar el desarrollo desde el genotipo hasta la expresión de su fenotipo [1].

El epigenoma no es estático, éste está influenciado por factores tanto internos como externos, se han implementado nuevos modelos que tratan de explicar el desarrollo humano donde se plantean además que el ambiente al que está expuesto una mujer embarazada interviene en su descendencia y que incluso este puede tener un impacto transgeneracional [2].

En la actualidad se requieren nuevas técnicas que permitan identificar individuos con riesgo de desarrollar alguna enfermedad, al momento existen indicadores clínicos como técnicas de imagen y biomarcadores que presentan algunas limitaciones. Por esta razón también se han desarrollado biomarcadores epigenéticos que han demostrado superar las técnicas de diagnóstico habituales y se espera que con el tiempo se incorporen a los estudios de rutina en diferentes áreas de la medicina [3].

\section{Metodología}

Diseño: El presente diseño se basó en una revisión que no cumple criterios meta analíticos.

Estrategia de búsqueda: En cuanto a criterios de inclusión, en esta investigación se marcó más interés en aquellos trabajos que mencionaban la epigenética influencia en el desarrollo de nuevas tecnologías para el desarrollo de marcadores clínicos predictivos de riesgo materno perinatal; los artículos publicados desde el año 2010, fueron los escogidos, destacando que solo se incluyeron artículos originales. A diferencia de los 
criterios de exclusión, los cuales se basaron en aquellos artículos que contaban con más variedades que no iban de acuerdo al tema, investigaciones que no poseían resultados e incluso artículos donde el texto no estaba disponible. Al preseleccionar los artículos, se hizo una búsqueda más amplia para apartar aquellos que se centraban en temas relevantes de epigenética, tecnología epigenética, marcadores clínicos predictivos, todo ello relacionado con riesgo materno perinatal, logrando conseguir artículos originales de relevancia, que, en su gran mayoría fueron experimentales.

Se procedió a realizar búsquedas en todas las bases de datos que a nivel mundial han tomado impacto, entre ellas están Elsevier, WoS, Pubmed, Scielo, Redalyc, entre otros, generando búsqueda con los términos epigenética, marcadores clínicos. Se realizó la búsqueda durante el tiempo transcurrido entre diciembre 2019-febrero 2020. El método de búsqueda consistió en utilizar como palabras clave en las diversas bases de datos: epigenética, marcadores clínicos predicativos, con la ayuda de conectores entre ellos 'and' y 'or', haciéndolo en singular y en plural; que los descriptores estén presentes en el resumen, título del artículo y, si la base de datos lo presenta, en palabras clave, estas palabras claves se buscaron en idioma español e inglés.

\section{Resultados}

Selección de estudios: Como se sabe que en cada base de datos las búsquedas son distintas se hizo de manera diferente en cada uno, y de los 37 artículos preseleccionados, descartamos 3 , pues los mismos no cumplían con los requerimientos de nuestra investigación, quedando así 34 artículos que si cumplían con los criterios inclusión que se había considerado.

\section{Discusión}

Epigenética: Estudia los complejos procesos que median entre el genotipo y el fenotipo, explicando por qué algunos organismos vivos expresan unos genes y silencian otros, fue descrito por primera vez por Waddingtonen en 1939 [1]. Una de las características del epigenoma es que no es estático por lo tanto puede modificarse, y es diferente en los distintos tejidos y células del organismo [4]. Al ocurrir modificaciones epigenéticas serán transmisibles de una generación a otra, es decir la descendencia portará los caracteres adquiridos por influencia tanto del ambiente como genéticamente [5].

Marcador clínico: Es una molécula biológica que podemos encontrar en la sangre, líquidos o tejidos que componen el cuerpo humano y ayuda como signo al verificar si está cursando por un proceso normal o anormal de alguna enfermedad. Puede servir además de eso para comprobar cómo responde el cuerpo a algún tratamiento que se esté realizando contra alguna patología. Es también llamado marcador molecular, biomarcador o molécula distintiva [6].

Factor de riesgo materno perinatal: Es cualquier rasgo, característica o exposición de una mujer embarazada que aumente la probabilidad de sufrir una enfermedad o 
lesión perinatal, entre los factores de riesgo materno perinatales cabe citar obesidad, hipertensión, diabetes, pielonefritis, entre otros [7].

Montealegre y Páez, en su artículo mencionan que a diario se amplían las investigaciones en el ámbito de salud, y que estas a la vez son herramientas que ayudan a mejorar la calidad vida de todas las personas actualmente, con los avances hechos en esta rama, se reconoce que existe una asociación de los estímulos extranucleares, extracelulares, ambientales y sociales en la modulación de los genes, siendo una parte fundamental en el estudio de las enfermedades que se exteriorizan durante el desarrollo precoz en asociación con eventos prenatales. Esto ha llevado a averiguar si existe mecanismos epigenéticos involucrados, buscando medidas de prevención y tratamientos a futuras enfermedades [8].

La revista de genética médica, señala que para poder analizar los marcadores clínicos debemos garantizar que las muestras estén perfectamente tomadas para un análisis sin errores, este es uno de los pasos más importantes y fundamentales, esto demanda un aislamiento eficiente y de alta calidad, tanto de la cromatina, histonas y ácidos nucleicos. Las muestras deben ser de un tamaño considerable, es decir, muestras grandes, que indiquen la zona que puede estar con alguna patología y la zona que se encuentra sana o sin presencia de esta, con lo cual se podría estudiar las células sanas para poder interponerse en las que tienen patología y revertir el proceso y evitar la enfermedad. Es necesario tener una tecnología de punta, que en el país no se dispone de manera fácil, por lo cual resulta ser una limitación muy grande, como por ejemplo, al analizar una muestra dependerá de que queremos observar, entonces se procederá, si se requiere analizar la metilación del ADN un tratamiento con bisulfito y si se requiere análisis del microADN circulante se procederá primero a un aislamiento y posterior una separación de exosomas [9].

Casanello, Krause, Castro y Uauy, indican en su artículo que la metilación del ADN es el principal marcador clínico que se puede evidenciar, este generalmente actúa silenciando genes mediante la agregación de un grupo metilo en el nucleótido de citosina que acompaña al de guanosina, que se encuentran en las regiones promotoras de el gen que vaya a ser modificado, por lo tanto las distintas enfermedades que aparecen en madres en etapa perinatal están relacionadas con estas mutaciones que van a cambiar el patrón de expresión del gen [10].

Paul Cordero, en su revisión refiere que los seres humanos según la etapa de vida en la que se encuentren, pueden sufrir de alteraciones en su epigenoma, pese a que puede darse en cualquier momento hay periodos en los cuales estos tienen más tendencia a incrementarse como es en el caso de la mujer embarazada, ya que se ve invadida por muchos cambios donde hay rápido y continua división celular lo que predispone a que el ADN este expuesto a cambios, interaccionar con el ambiente, hormonas, nutrientes, entre otras presentes en el contexto intrauterino. Realizar estudios en mujeres embarazadas ha sido complicado debido a que la ética nos limita, pues el personal de salud no acepta realizar intervenciones que no se sepa con certeza y seguridad si van a resultar beneficiosas en esta etapa; y por otro lado resulta demoroso ya que para estos estudios se los debe llevar a largo plazo [11]. 
Taucher Castillo, describe en su revisión, los riesgos en madres que cursan el periodo perinatal van a depender de muchos factores como son el peso, la altura, el número de embarazos o abortos anteriores, uso de anticonceptivos, historial de operaciones, edad, enfermedades previas, sangrados vaginales, accidentes, uso de anestesia, duración del parto, uso de fórceps, estrés, ansiedad, depresión, relación con la pareja, entre muchos más, a los cuales debemos prestar mucha atención y enfocarse en aquellos que merecen más atención. Algo favorable según estudios epigenéticos es la lactancia materna que ayuda en el desarrollo de la microbiota materna y microbiota neonatal, por ello es de interés para los equipos de salud perinatal que, quienes realicen este control sean profesionales capacitados en perinatología para el beneficio del binomio madre hijo y con ello de futuras generaciones, ya que se convierten en un ser privilegiado que tiene una posición de asesor para la protección contra dichas alteraciones epigenéticas [12], mientras que Keklikián y Coronel por su parte comentan que existen factores a prestar más atención para su identificación y en el mejor de los casos su eliminación antes de que las mismas realicen cambios epigenéticos y generen consecuencias patológicas. Algunos autores mencionan en sus estudios que los cambios en los mecanismos epigenéticos que se producen en el embarazo, ocurren siempre y cuando el ambiente intrauterino sea desfavorable, y estos factores son: Determinantes sociales vulnerables, el estrés materno, la desnutrición, el uso de sustancias psicotrópicas como drogas, la violencia, la exposición a hormonas debido a los cambios endocrinos, la hipoxia como también las enfermedades que la madre [13].

Martínez y Miranda en otra importante investigación [14, 15] denota que se han desarrollado nuevas formas de inserción de la epigenética en este arduo estudio como la demostración de la correlación nutricional materna con la metilación de ADN, con la modificación de ARN e histonas, asociándose al desarrollo de obesidad y diabetes; al igual, la interacción del eje intestino cerebro puede llegar afectarse dando paso a otras enfermedades psíquicas como depresión, ansiedad siendo que el 10\% de las enfermedades crónicas pueden deberse a factores epigenéticos.

En un reporte de gastroenterólogos también se menciona a la diabetes pues es uno de los problemas a los que también se ven afectadas las madres en su periodo de embarazo y perinatal, estudios epigenéticos han demostrado cuales son los genes que sufren cambios ante esta patología, tomando en cuenta que aún no se han comprobado todos los genes que están involucrados, dos de ellos son el gen SLC2A4 y el gen $A B C C 8$, el primero por deficiencias calóricas que codifica la proteína transportadora de glucosa regulada por la insulina, es decir el GLUT4 y el segundo que promueve la liberación de la insulina a partir de células beta pancreáticas [16].

La revisión de Milagro y Martínez, en cuanto a la obesidad, estudios han dado a conocer que esto se centra más en la alimentación de la madre tanto en la gestación como en la etapa de lactancia, e incluso el estrés, que también resulta de la metilación del ADN promotor del receptor de glucocorticoides, que es clave pues regula la adiposidad corporal, que mayormente se enfoca en la zona visceral, secundario a estrés materno, bajo cuidado infantil posparto o también abuso infantil, por lo tanto estas personas tienen mayor respuesta al estrés que puede presentarse de manera física o psíquica [17]. Acotando con ello otro estudio epigenómico en personas obesas 
aborda que se identifican células con sitios de ADN metilado que se asocia a esta patología y que después de someterse a una dieta, ejercicio físico o una combinación de ambos, estos estudios siguen siendo los mismos sin generar ningún cambio ni revisión; contrarrestando con las mujeres embarazadas normo peso que tras terminar esta etapa pueden retomar sus medidas iniciales [18].

Para Adrián C., una de las complicaciones obstétricas que suelen presentarse muy seguido es la preeclampsia, la misma que puede darse de dos maneras: Sintomática y no sintomática, esto resulta al producirse modificaciones de la cromatina epigenética placentaria en particular la acetilación de las histonas y la metilación del ADN. Por lo tanto al existir un ambiente intrauterino alterado, el crecimiento fetal también se verá alterado, generándose cambios en la estructura de la cromatina, alterando la unión de los factores de la transcripción y así puede dar como resultado daño vascular que puede terminar en hipoxia, ocasionando daños potencialmente mortales y de emergencia [19].

Algunos artículos [20-22] mencionan que el maltrato materno produce en los niños una respuesta alterada al estrés, esto se debe a fallas en el eje hipotálamo-hipófisisadrenal ( $\mathrm{HHA}$ ) encontrándose que esta alteración se debe a metilación del gen NR3C1, siendo este sensible al estado anímico de las madres en periodo prenatal. Tanto las madres como sus productos son susceptibles al mínimo cambio en su comportamiento natural y uno de esos cambios drásticos es el tener dentro de su cuerpo la presencia de un ser y tras el nacimiento, la usencia del mismo, a lo cual su organismo le va a resultar algo extraño y puede generarse depresión.

La carta al editor de Landires y Núñez señala que es muy curioso entender que la mayoría de las células con sus expresiones son heredadas de generación en generación, sin embargo, las células de la memoria son independientes de cada individuo es decir no son heredadas de ninguno de nuestro antepasados; el conocimiento de las nuevas líneas de investigación en epigenética avanzan de forma rápida y brindan esperanza para tratar posteriormente enfermedades de origen genético y entender cómo se involucra el ambiente en el desarrollo de las mismas [23].

Varios artículos $[18,24]$ refieren que de la mano con el periodo prenatal existe correlación que eventos post-natales pueden influir en el desarrollo de enfermedades a edades precoces y a la exacerbación de enfermedades crónicas en la vida adulta. La calidad de vida y un mal desarrollo del vínculo materno se han asociado a un aumento de la respuesta endocrina en la vida adulta como alteraciones a las respuestas de estímulos estresantes, lo que concuerda con las observaciones en animales. Según Casanello et al. la epigenética actualmente es una ciencia que avanza despacio, la observación de los cambios genéticos por estrés ambiental externo a la célula, como el desarrollo de biomarcadores prenatales para la detección de enfermedades es aún el paso a seguir dando. Este nuevo desafío nos plantea la creación de nuevos avances cibernéticos y metodológicos [18].

Una revisión bibliográfica refiere que está claro que existen muchos riesgos a los que nos enfrentamos a diario al padecer o no alguna enfermedad y una mujer en edad fértil, embarazada o puérpera también los tendrá, y estos pueden ser diferentes ya que cada una de ellas es un ser humano distinto; estudios realizados en diversos lugares demostraron que pese a si la madre tiene o no una buena nutrición, el organismo del 
nuevo ser, se prepara para enfrentarse a un entorno nutricional adverso al que tiene intrauterino, adicional a que si la madre padeció hambruna posteriormente pueden desarrollar enfermedades cardiovasculares, diabetes y dislipidemias [25].

Se han evidenciado cuatro etapas muy significativas en las cuales se puede realizar acciones para producir cambios epigenéticos: En el periodo de fertilización y la formación del blastocisto, durante la gastrulación, en la organogénesis fetal y nacimiento de producto y también las intervenciones que se realizan en todo el proceso [13].

Penchaszadeh menciona que algunos de los adelantos recientes en epigenética incluyen el conocimiento de toda la expresión genética del individuo, que la cromatina es el receptor de todos los estímulos e influencias ambientales que tienden a producir cambios en el ADN dentro de los cuales también incluyen la transcripción, reparación y replicación; las histonas que tienen capacidad de leer las modificaciones epigenéticas ya realizadas en la cromatina y pueden incorporarlas o borrarlas del genoma y también que estos mecanismos epigenéticos pueden ser reprogramados, por lo cual es importante realizar estudios certeros sin tendencia a la equivocación. Se ha creado un mapa del epigenoma lo cual resulta beneficioso para poder realizar estudios más rápidos ya conociendo bien como es una célula en un estado natural sin presentar alteraciones, y al tener por otro lado una célula con alteraciones que da paso al desarrollo de alguna enfermedad [26].

Pata Aldhous, Hor y Reynolds, una buena dieta, es decir equilibrada de acuerdo a las características propias de cada individuo durante la etapa del embarazo, es imprescindible tanto para el producto como para la madre. Por otro lado, también se sugiere que la nutrición paterna también podría tener un efecto que produzcan cambios epigenéticos en la esperma que repercutan en futuras generaciones [27]. Cordero también concuerda que al saber que somos el resultado de lo que comemos y de lo que comieron nuestras madres, el peso adecuado, la dieta y los estilos de vida saludables, en las etapas del embarazo y la lactancia favorecerán para un correcto desarrollo de la descendencia [11].

La revisión bibliográfica de Milagro y Martínez [28] alude que el futuro de la epigenética es muy impreciso pero a ciencia cierta traerá muchos beneficios para la salud de toda la población en general y con especial énfasis en las mujeres embarazadas. Una base fundamental resultará la nutriepigenética debido a que la nutrición siempre debe ser personalizada, y al demostrar alguno de los cambios epigenéticos que pueden ocurrir se podrán implementar tratamientos dietéticos (dieta epigenética), retrasando o interviniendo en dichos cambios y evitando el desarrollo de enfermedades metabólicas.

Para Sun et al. otra de las bases de la epigenética va a ser la farmacoepigenética, para intervenir con tratamientos farmacológicos personalizados, sin embargo llevara más tiempo al tener que comprobar la respuesta a los mismos y demostrar que se revierten las modificaciones epigenéticas que ya se hayan producido [29].

Mas, Thu, Maluf en su artículo plantean que en un futuro se pretenden realizar trasplantes de órganos que hayan desarrollado alguna patología después de realizar estudios a sus donantes para asegurar que su epigenoma no se encuentre alterado, con el fin de que el huésped no reaccione y rechace los mismos [30]. La Red de Excelencia de Epigenoma menciona que en el caso de que el paciente necesite de 
un órgano nuevo gracias a la epigenética y a su combinación el uso de células madre del organismo se puede conseguir que las mismas proliferen y se desarrollen. Algunas células tienen los genomas cerrados en ciertas regiones y al poder intervenir en estos se podrá lograr su diferenciación ya que el ADN siempre está abierto a recibir y ejecutar órdenes pese a que sean manipulaciones. Esto suena muy interesante pero aun cuesta descifrar como lograr que las células ejecuten estas instrucciones, más aun hablando de ética, equipo técnico y práctico e incluso la financiación de esto [31].

Para Alonso et al. entre las recomendaciones encontradas en diferentes artículos científicos que se han ido revisando se menciona que los estudios epigenéticos se los podría realizar a madres que desean un embarazo, puesto que esto también sería beneficioso y los resultados que se arrojarían ayudarían a relacionar la prevalencia de la presencia o no de ciertas patologías siendo así, prueba diagnóstica relevante cuando esta muestra algún patrón predictor, moderada cuando la muestra resulta poco consistente y baja cuando la misma es incierta, por lo tanto en las primeras se podría intervenir un cierto tiempo antes de que la paciente entre en etapa de embarazo, mejorando las probabilidades tanto para ella como para la del producto. Por lo tanto aún siguen abundando distintas líneas de investigación que avanzan rápidamente en la búsqueda de respuestas en especial en aquellos países donde tanto la tecnología da pasos agigantados y con ello la medicina, brindando la esperanza al personal de salud de que la epigenética es el futuro para tratar enfermedades que se presentan por cambios genéticos o ambientales [32].

Como se puede percatar realizar estudios de epigenética no resulta tan sencillo como parece, todo lo que se ha logrado hasta el día de hoy resulta del esfuerzo tan arduo y constante que tienen las personas para buscar el bien de la sociedad, minimizando los factores de riesgo que puedan cursar a lo largo del desarrollo de una patología, debido a que si una persona se encuentra con alguna patología no solo repercute en ella si no en su entorno, es muy importante seguir avanzando en la búsqueda de información, que si bien es cierto toma tiempo y conlleva mucha voluntad, ganas, energía, entre otras cosas; nuestro país no resulta ser uno de los primeros en generar investigaciones, por características como son la falta de financiamiento, la falta de laboratorios bien equipados, los instrumentos y recursos y lo más importante la población para realizar estos estudios, que muchas veces por miedo al que pasara no se atreven y como ya se lo mencionó anteriormente es un tema de ética médica con lo cual no se puede jugar fácilmente, sin embargo actualmente los centros más nombrados en Ecuador son El Instituto Nacional de Investigación en Salud Pública Dr. Leopoldo Izquieta Pérez (INSPI) y el Centro de Investigación de Genética y Genómica (CIGG) de la Universidad Tecnológica Equinoccial (UTE), donde este último es el primer laboratorio del país dedicado al estudio de genes y genomas del ADN de los ecuatorianos [33].

Es por eso que los países como son Estados Unidos, España, Alemania, Corea del Sur, entre otros, que cuentan con mucha más tecnología ya han realizado estudios de epigenética cuyos resultados hacemos uso en medicina, son el Programa del Mapa Epigenómico que los Institutos Nacionales de Salud ( $\mathrm{NIH}$, según sus siglas en inglés) de Estados Unidos, el Centro Nacional de Análisis Genómico (CNAG), International Human Epigenome Consortium (IHEC) y el que más se destaca en realizar estas investigaciones 
es el proyecto BLUEPRINT, que menciona que el genoma es el hardware y el epigenoma es el software en una programación. Con la información que hasta el día de hoy se tiene la cual es muy impactante para proceder a realizar un tratamiento o intervención en general, al pensar que no se pueden realizar estos estudios en un laboratorio convencional, lo que se pretende es diseñar unos kits que aporten a generar resultados fiables al tener algún predictor de enfermedad, en especial en las personas que son prioridad como son las madres en etapa perinatal [34].

\section{Conclusiones}

Con el paso de los años la medicina ha ido avanzando como también lo ha hecho la tecnología, el poder actual de la epigenética, nos da pautas para tener una explicación del porqué ocurren un sin número de patologías; el poder enfocarse en evaluar los riesgos materno perinatales es de gran ayuda, al demostrar qué marcadores clínicos están o no presentes. Para realizar estas investigaciones es necesario tener tecnología de punta que no se puede encontrar en cualquier lugar, si no en países del primer mundo pues las muestras, también deben tener un protocolo que cumplir, la metilación del ADN es el principal marcador clínico con el que se realizan las investigaciones. La mujer, se ve mucho más comprometida en la etapa de embarazo ya que experimenta una serie de cambios que le predisponen al desarrollo de alguna patología, las investigaciones en ellas se ven limitadas por el mismo hecho de tener una vida formándose dentro de ellas, pero las enfermedades que resaltan son la diabetes, obesidad y la preeclampsia donde la epigenética ha demostrado que suceden cambios en genes específicos. El objetivo que se busca conseguir es detectar de manera más temprana con ayuda de tecnología adecuada, marcadores clínicos que alerten a prestar más atención antes de que se produzca algún daño para la madre y por consiguiente lograr que sea una etapa plena para la mujer y el producto.

\section{Agradecimientos}

Todo agradecimiento se lo debemos a la Facultad de Salud Pública, de la Escuela Superior Politécnica de Chimborazo, al realizar el XVIII Seminario Internacional de Salud, Alimentación, y Nutrición Humana, a la vez al comité organizador, por todo el esfuerzo en la difusión de investigación científica.

\section{Conflictos de Interés}

Los autores de este artículo declaramos no tener ningún conflicto de interés.

\section{Limitación de Responsabilidad}

Todos los enfoques mencionados en la presenta revisión bibliográfica son de completa responsabilidad de los autores. 


\section{Fuentes de Apoyo}

Propias del grupo de autores de este artículo.

\section{References}

[1] Bedregal P, Shand B, Santos MJ, Ventura-Juncá P. Aportes de la epigenética en la comprensión del desarrollo del ser humano. Revista Medica de Chile. 2010;138(3):366-72.

[2] Casanova AR. Epigenética y desarrollo humano. La Habana; 2013. Available from: http://www.cbioetica. org/revista/133/133-1525.pdf

[3] Andersen AM, Dogan M V., Beach SRH, Philibert RA. Current and future prospects for epigenetic biomarkers of substance use disorders. Genes (Basel). 2015;6(4):991-1022.

[4] Teherán M. Epigenética un mundo de cambios y modificaciones. Revista Salud en Movimiento. 2017 [citado 19 de febrero de 2020]. Disponible en: http://publicaciones.unisimonbolivar.edu.co/rdigital/ojs/ index.php/saludmov/article/view/1627/1503

[5] Anastasiadi D, Esteve-Codina A, Piferrer F. Consistent inverse correlation between DNA methylation of the first intron and gene expression across tissues and species. Epigenetics Chromatin. 2018;11(1):37.

[6] Definición de marcador biológico- Diccionario de cáncer. - National Cancer Institute. Available from: https://www.cancer.gov/espanol/publicaciones/diccionario/def/marcador-biologico

[7] Artal R. Factores de riesgo para complicaciones durante el embarazo - Ginecología y obstetricia - Manual MSD versión para profesionales. 2017. Available from: https: //www.msdmanuals.com/es/professional/ginecología-y-obstetricia/embarazo-de-alto-riesgo/ factores-de-riesgo-para-complicaciones-durante-el-embarazo

[8] Montealegre L. Investigación en salud materno-perinatal. Rev Salud Bosque. 2017 [citado 19 de febrero de 2020]; Disponible en: https://revistasaludbosque.unbosque.edu.co/article/view/2189/1719

[9] García J, Pérez G, Beltrán J. Genética médica y genómica. 2018. Biomarcadores epigenéticos: hacia su implantación en la rutina clínica. p. 45.

[10] Casanello P, Krause BJ, Castro-Rodriguez JA, Uauy R. Fetal programming of chronic diseases: Current concepts and epigenetics. Rev Chil Pediatr. 2015;86(3):135-7.

[11] Cordero P. Obesidad, nutrición perinatal y epigenética. Revista Matronas. 2014 [citado 17 de febrero de 2020]. Disponible en: https://www.enfermeria21.com/revistas/matronas/articulo/30/ obesidad-nutricion-perinatal-y-epigenetica/

[12] Taucher CS. Consideraciones sobre epigenética perinatal. Bol Hosp Viña del Mar. 2015;19-22.

[13] Keklikián R, Coronel A. Microbioma y Epigenética en Perinatología: 1000 días que pueden cambiar destinos. Available from: http://www.fasgo.org.ar/images/1000diasmicrobiomaepigenetica.pdf

[14] Martínez A, Martínez O. Nutrición y obesidad: Aplicaciones "ómicas" [Internet]. 2017. Available from: http://renhyd.org/index.php/renhyd/article/view/484

[15] Miranda V. Relación entre microbiota intestinal, epigenética y exposoma en la salud materno infantil. Revista Cubana de Pediatría. 2019 [citado 19 de febrero de 2020]. Disponible en: http://scielo.sld.cu/ pdf/ped/v91n2/1561-3119-ped-91-02-e786.pdf

[16] Sun X, Li P, Yang X, Li W, Qiu X, Zhu S. From genetics and epigenetics to the future of precision treatment for obesity. Gastroenterol Rep. 2017;5(4):266-70.

[17] Milagro F, Martínez A. Epigenética en obesidad y diabetes tipo 2: papel de la nutrición, limitaciones y futuras aplicaciones. 2013. Available from: http://www.revistasoched.cl/3-2013/3-2013.pdf\#page=22

[18] Casanello P, Krause BJ, Castro-Rodríguez JA, Uauy R. Epigenética y obesidad. Rev Chil Pediatr 2016;87(5):335-42.

[19] Eddy AC, Chapman H, George EM. Acute hypoxia and chronic ischemia induce differential total changes in placental epigenetic modifications. Reprod Sci. 2019;26(6):766-73.

[20] Parra-Ruiz C, Prado G, Cerda D, et al. Obesidad parental y modificaciones epigenéticas en la descendencia. Rev Chil Nutr. 2019;46(6):792-9.

[21] Girchenko P, Lahti J, Czamara D, et al. Associations between maternal risk factors of adverse pregnancy and birth outcomes and the offspring epigenetic clock of gestational age at birth. Clin Epigenetics. 2017;9(1):49.

[22] Reid MA, Dai Z, Locasale JW. The impact of cellular metabolism on chromatin dynamics and epigenetics. Nature Cell Biology. 2017;19:1298-306.

[23] Landires I, Núñez-Samudio V. Epigenetics, memory, and inheritance. Neurologia. 2019;34: mar [citado 17 de febrero de 2020]. Disponible en: https://www.elsevier.es/ 
es-revista-neurologia-295-articulo-epigenetica-memoria-herencia-S0213485317301597?referer= buscador

[24] Chandak GR, Silver MJ, Saffari A, et al. Protocol for the EMPHASIS study; epigenetic mechanisms linking maternal pre-conceptional nutrition and children's health in India and Sub- Saharan Africa. BMC Nutr. 2017;3(1).

[25] Marín. Marvelis de la Rosa, Ledea L, Ferrera Y, Laffita A. Epigenetica, desarrollo intrauterino y síndrome metabolico. Revision Bibliogtafica. Morfovirtual 2018. 2018 [citado 17 de febrero de 2020]; Disponible en: http://www.morfovirtual2018.sld.cu/index.php/morfovirtual/2018/paper/viewPaper/277/580

[26] Penchaszadeh V. Mecanismos epigenéticos y mecanismos epigenéticos y programación fetal. 2018 [citado 17 de febrero de 2020]. Disponible en: https://www.sap.org.ar/docs/congresos_2018/DOHAD/ DO01-DOHAD-Oport_en_etapas_criticas-Penchaszadeh.pdf

[27] Aldhous MC, Hor K, Reynolds RM. Handbook of Nutrition and Pregnancy. Cham: Springer International Publishing; 2018. Epigenetics and diet in pregnancy; p. 163-81.

[28] Fermín M, Martínez A. La nutrición personalizada a través de la epigenética. Mediterráneo económico. 2008;(13):43-54.

[29] Sun X, Li P, Yang X, Li W, Qiu X, Zhu S. From genetics and epigenetics to the future of precision treatment for obesity. Gastroenterol Rep. 2017;5(4):266-70.

[30] Mas VR, Le TH, Maluf DG. Epigenetics in kidney transplantation. Transplantation. 2016;100(1):23-38.

[31] Epigenética. La epigenética y el futuro de las células madre. Epigenética. Available from: http: //epigenome.eu/es/2,8,613.html

[32] Alonso-Cerezo MC, Calero Ruiz M, Chantada-Abal V, et al. Recommendations regarding the genetic and immunological study of reproductive dysfunction. Med Clin (Barc). 2018;151(4):161.e1-161.e12.

[33] Veletanga J. La UTE tiene el primer Centro de Investigación de Genética y Genómica. Edición médica. 2016. Available from: https://www.edicionmedica.ec/secciones/gestion/ ute-presenta-nuevo-centro-de-investigaci-n-de-gen-tica-y-gen-mica-89057

[34] López Á. La sala de máquinas del genoma. Madrid; 2015. Available from: https://www.irbbarcelona. org/sites/default/files/news/2015/02/2015_02_19_elmundo_epigenetica_fazorin.pdf 